\title{
A Multi-domain Spectral Method for Time-fractional Differential Equations
}

\author{
Feng Chen ${ }^{\mathrm{a}}$, Qinwu $\mathrm{Xu}^{\mathrm{b}}$, Jan S Hesthaven ${ }^{\mathrm{c}}$ \\ ${ }^{a}$ Division of Applied Mathematics, Brown University, Providence, RI 02912, USA Email: feng_chen_1@brown.edu \\ ${ }^{b}$ School of Mathematics and Statistics, Central South University, China. Email: qw_xu@hotmail.com \\ ${ }^{c}$ EPFL-SB-MATHICSE-MCSS, École Polytechnique Fédérale de Lausanne (EPFL), Switzerland. Email: jan. hesthaven@epfl.ch
}

\begin{abstract}
This paper proposes an approach for high-order time integration within a multi-domain setting for timefractional differential equations. Since the kernel is singular or nearly singular, two main difficulties arise after the domain decomposition: how to properly account for the history/memory part and how to perform the integration accurately. To address these issues, we propose a novel hybrid approach for the numerical integration based on the combination of three-term-recurrence relations of Jacobi polynomials and high-order Gauss quadrature. The different approximations used in the hybrid approach are justified theoretically and through numerical examples. Based on this, we propose a new multi-domain spectral method for high-order accurate time integrations and study its stability properties by identifying the method as a generalized linear method. Numerical experiments confirm $h p$-convergence for both time-fractional differential equations and time-fractional partial differential equations.
\end{abstract}

Keywords: multi-domain, spectral, time-fractional, high-order integration, three-term-recurrence, general linear method.

\section{Introduction}

Fractional calculus and the modeling of a variety of non-classic phenomena using fractional differential equations is emerging as an area of substantial activity across many applications in the natural and social sciences (cf. $[17,16])$. The primary advantage of such models is the introduction of a parameter, $\alpha$, which can be used to model non-Markovian behavior of spatial or temporal processes. During the last decade, this approach has emerged as generalizations of many classic problems in mathematical physics, including the fractional Burgers' equation [11, 25], the fractional Navier-Stokes equation [7, 6], the fractional Maxwell equation [10], the fractional Schrödinger equation [8], the fractional Ginzburg Landau equation [8, 22], etc. In parallel, numerical methods for classical differential equations, such as finite difference methods [15, 14, 23], finite element methods [3], spectral methods [13, 2, 12], and discontinuous Galerkin methods $[19,24]$, have been developed, albeit this remains a relatively new topic of research.

In this work we focus on time-fractional differential equations (TFDE) where the time-fractional derivative emerges as an integro-differential operator, defined by the convolution of the classical derivative of the function and a singular kernel of fractional power-law type. Hence, the solution to a TFDE at a certain time depends on the total history of the solution at previous times and seek to model problems with memory terms, among other things. Considering the design of numerical schemes, an immediate 
consequence of this is that the whole trajectory of the numerical solution must be carried forward and used in the computation at the current time step. This impacts both the storage and the cost of the numerical method, both of which may substantially increase over time.

Most past work on the development of methods for TFDEs has focused on lower order methods in which a finite difference approach is used to replace the integrant with its forward, backward, or mid-point rules. More recently, a piecewise-linear, discontinuous Galerkin method was proposed in [19] for the fractional wave equation.

Though only sparsely explored in this context, high order methods have the potential to reduce the storage requirement and computational cost, by allowing the use of many fewer points while achieving the same accuracy as that of lower order methods. However, accurate high-order approximation to fractional operators requires a delicate treatment of special functions and integral transforms, similar to what has been developed in the context of spectral methods based on classic orthogonal polynomials $[9,5]$. Using spectral methods for the TFDE, [13] proposes a spectral-Galerkin method and shows that by taking advantage of special properties of the time-fractional differential operator, positive definite linear systems result, leading to an efficient solver. In [2], the authors explored analytical results of the fractional derivative of shifted Jacobi polynomials and discussed two effective approaches - spectral collocation methods and spectral tau methods - for the fractional differential equation. Recently, [12] derived three-term-recurrence relations for fractional integrals and derivatives for the Jacobi polynomials, and provided an elegant collocation approach that can be seen as a generalization of the classical numerical differentiation. A slightly different but related approach is developed in [26] where a Petrov-Galerkin spectral element method is proposed for fractional ODEs, defined through the Riemann-Liouville definition. Unlike [26], in this paper we focus on a collocation form that is more suitable for variable coefficients and nonlinear source functions.

The majority of past developments of high-order methods for the TFDE emphasize spectral approximations in a single domain. In this paper, we adopt the idea of a multi-domain spectral approach, leading to a generalized high-order linear method with a long tail of past solutions. A similar approach is recently proposed in There are two challenges that arise out of this approach. First, one needs to discretize the fractional derivative which has a singular kernel within the current element. For this, we rely on the use of the three-term-recurrence approach proposed in [12]. The second task, which appears more challenging, is to compute the history part accurately and efficiently, i.e., to compute the integral over previous elements with high precision. In order to deal with this, we first extend the three-term-recurrence relation in [12] to work on all elements and then combine it with a Gaussian quadrature for terms far away from the current element. This hybrid approach is needed to control stability issues related to the recurrence relation. Values of these integrals together with the source function form the right hand side of the linear system.

To understand the stability properties of this approach, we carry out a stability study based on a companion matrix approach and verify $h p$-convergence of our method for time-fractional differential equations and time-fractional partial differential equations. The paper seeks to establish a computational framework for high-order accurate integration of the TFDE, and our work clearly indicates that the multi-domain spectral approach, as an optimized combination of low and high order methods, can provide excellent performance for numerical simulations of problems dominated by memory effects.

What remains of the paper is organized as follows. Basic notation of fractional calculus and TFDE are provided in Section 2. In Section 3, we describe the core techniques of our spectral approximation to the fractional derivative. The algorithmic details and the stability analysis of the hybrid multi-domain spectral method are discussed in Section 4. Numerical results for a multi-term fractional differential equation are 
provided in Section 5 and Section 6 contains a few concluding remarks.

\section{Fractional differential equations}

Let us denote the time domain $(0, T)$ as $\Omega$, and let $t \in \Omega$. The fractional integral of order $\alpha$ of a given function $u(t)$ is defined as

$$
{ }_{0} D_{t}^{-\alpha} u(t) \triangleq \frac{1}{\Gamma(\alpha)} \int_{0}^{t}(t-s)^{\alpha-1} u(s) \mathrm{d} s,
$$

where $\Gamma(x)$ is the Gamma function. This allows us to define the Caputo fractional derivative of order $\alpha,(0<\alpha \leq 1)$ as

$$
{ }_{0} D_{t}^{\alpha} u(t) \triangleq \frac{1}{\Gamma(n-\alpha)} \int_{0}^{t}(t-s)^{n-1-\alpha} \frac{\mathrm{d}^{n} u(s)}{\mathrm{d} s^{n}} \mathrm{~d} s .
$$

which is preferred over alternative definitions to deal with general initial conditions. This definition can naturally be extended to higher order as

$$
{ }_{0} D_{t}^{\alpha} u(t) \triangleq{ }_{0} D_{t}^{\alpha-n} \frac{\mathrm{d}^{n} u(t)}{\mathrm{d} t^{n}}=\frac{1}{\Gamma(n-\alpha)} \int_{0}^{t}(t-s)^{n-1-\alpha} \frac{\mathrm{d}^{n} u(s)}{\mathrm{d} s^{n}} \mathrm{~d} s,
$$

where $n$ is an integer such that $\alpha \in(n-1, n]$.

With this notation we express the multi-term fractional differential equation is written as:

$$
\left\{\begin{array}{l}
\sum_{k=1}^{m} a_{k}(t){ }_{0} D_{t}^{\alpha_{k}} u(t)=\mathcal{L} u(t)+f(t), \quad t \in \Omega, \\
u^{(k)}(0)=u_{k}, \quad 0 \leqslant k \leqslant n-1 .
\end{array}\right.
$$

Here we assume that $0 \leqslant \alpha_{1}<\alpha_{2}<\cdots<\alpha_{m}$ and $\alpha_{m} \in(n-1, n]$. On the right hand side of the equation, $\mathcal{L}$ can be either a scalar function for the time-fractional differential equation (TFDE) case or an operator in a spatial domain for the time-fractional partial differential equation (TFPDE) case. We refer to [20] for a general background on the fractional calculus.

\section{Fractional calculus using spectral methods}

In the first part of this section, we provide the required background on Jacobi polynomials and fundamentals of spectral approximations to fractional derivatives in the single domain. This sets the stage for the second part, discussing key ideas related to the fractional derivative of Jacobi polynomials in a multi-element setting.

\subsection{Spectral approximations in the single domain}

We begin by recalling basic properties of Jacobi polynomials, and then describe how to construct differentiation matrices for fractional differentiation using three term relations of Jacobi polynomials and their fractional derivatives. 


\subsubsection{Jacobi polynomials}

Denote $J_{j}^{a, b}(t)$ as the $j$-th order Jacobi polynomial of index $(a, b)$ defined on $[-1,1]$. As all classic orthogonal polynomials, $\left\{J_{j}^{a, b}(t)\right\}_{j=0}^{N}$ satisfies the following three-term-recurrence relation (cf. [21, 5]):

$$
\begin{aligned}
& J_{0}^{a, b}(t)=1, \\
& J_{1}^{a, b}(t)=(a+b+2) t+(a-b), \\
& J_{j+1}^{a, b}(t)=\left(A_{j}^{a, b} t-B_{j}^{a, b}\right) J_{j}^{a, b}(t)-C_{j}^{a, b} J_{j-1}^{a, b}(t), \quad 1 \leqslant j \leqslant N-1,
\end{aligned}
$$

with the recursion coefficients given as

$$
\begin{aligned}
A_{j}^{a, b} & =\frac{(2 j+a+b+1)(2 j+a+b+2)}{2(j+1)(j+a+b+1)}, \\
B_{j}^{a, b} & =\frac{\left(b^{2}-a^{2}\right)(2 j+a+b+1)}{2(j+1)(j+a+b+1)(2 j+a+b)}, \\
C_{j}^{a, b} & =\frac{(j+a)(j+b)(2 j+a+b+2)}{(j+1)(j+a+b+1)(2 j+a+b)} .
\end{aligned}
$$

The endpoint values of the Jacobi polynomial are given as

$$
J_{j}^{a, b}(-1)=(-1)^{j}\left(\begin{array}{c}
j+b \\
j
\end{array}\right), \quad J_{j}^{a, b}(1)=\left(\begin{array}{c}
j+a \\
j
\end{array}\right) .
$$

We also recall the relation between the Jacobi polynomial and its derivative as

$$
\frac{\mathrm{d}^{m}}{\mathrm{~d} x^{m}} J_{j}^{a, b}(t)=d_{j, m}^{a, b} J_{j-m}^{a+m, b+m}(t), \quad m \geqslant j, \quad m \in \mathbb{N},
$$

where

$$
d_{j, m}^{a, b}=\frac{\Gamma(j+m+a+b+1)}{2^{m} \Gamma(j+a+b+2)} .
$$

Finally, we recall that a Jacobi polynomial can be written as a sum of derivatives on the form

$$
J_{j}^{a, b}(t)=\bar{A}_{j}^{a, b} \frac{\mathrm{d}}{\mathrm{d} t} J_{j-1}^{a, b}(t)+\bar{B}_{j}^{a, b} \frac{\mathrm{d}}{\mathrm{d} t} J_{j}^{a, b}(t)+\bar{C}_{j}^{a, b} \frac{\mathrm{d}}{\mathrm{d} t} J_{j+1}^{a, b}(t),
$$

where the coefficients are defined as

$$
\begin{aligned}
\bar{A}_{j}^{a, b} & =\frac{-2(j+a)(j+b)}{(j+a+b)(2 j+a+b)(2 j+a+b+1)}, \\
\bar{B}_{j}^{a, b} & =\frac{2(a-b)}{(2 j+a+b)(2 j+a+b+2)}, \\
\bar{C}_{j}^{a, b} & =\frac{2(j+a+b+1)}{(2 j+a+b+1)(2 j+a+b+2)} .
\end{aligned}
$$




\subsubsection{Fractional differentiation matrix using three-term-recurrence relations}

We recall the following result [12]

Theorem 3.1. Let the fractional integral of Jacobi polynomials be defined as

$$
\hat{J}_{j}^{a, b, \alpha}(t):={ }_{-1} D_{t}^{-\alpha} J_{j}^{a, b}(t)=\frac{1}{\Gamma(\alpha)} \int_{-1}^{t}(t-s)^{\alpha-1} J_{j}^{a, b}(s) \mathrm{d} s .
$$

Then $\left\{\hat{J}_{j}^{a, b, \alpha}(t)\right\}_{j=0}^{N}$ satisfies the following three-term-recurrence relation:

$$
\begin{aligned}
& \hat{J}_{0}^{a, b, \alpha}(t)=\frac{(t+1)^{\alpha}}{\Gamma(\alpha+1)} \\
& \hat{J}_{1}^{a, b, \alpha}(t)=\frac{a+b+2}{2}\left(\frac{t(t+1)^{\alpha}}{\Gamma(\alpha+1)}-\alpha \frac{(t+1)^{\alpha+1}}{\Gamma(\alpha+2)}\right)+\frac{a-b}{2} \hat{J}_{0}^{a, b, \alpha}(t), \\
& \hat{A}_{j}^{a, b} \hat{J}_{j+1}^{a, b, \alpha}(t)=\hat{B}_{j}^{a, b} \hat{J}_{j}^{a, b, \alpha}(t)+\hat{C}_{j}^{a, b} \hat{J}_{j-1}^{a, b, \alpha}(t)+\hat{D}_{j}^{a, b}(t+1)^{\alpha},
\end{aligned}
$$

where

$$
\begin{aligned}
\hat{A}_{j}^{a, b} & =\left(1+\alpha A_{j}^{a, b} \bar{C}_{j}^{a, b}\right), \\
\hat{B}_{j}^{a, b} & =\left(A_{j}^{a, b} x-B_{j}^{a, b}-\alpha A_{j}^{a, b} \bar{B}_{j}^{a, b}\right), \\
\hat{C}_{j}^{a, b} & =\left(C_{j}^{a, b}+\alpha A_{j}^{a, b} \bar{A}_{j}^{a, b}\right), \\
\hat{D}_{j}^{a, b} & =\frac{A_{j}^{a, b}}{\Gamma(\alpha)}\left(\bar{A}_{j}^{a, b} J_{j-1}^{a, b}(-1)+\bar{B}_{j}^{a, b} J_{j}^{a, b}(-1)+\bar{C}_{j}^{a, b} J_{j+1}^{a, b}(-1)\right) .
\end{aligned}
$$

Using (3.3), the fractional derivative of a Jacobi polynomial can be expressed as

$$
\left(J_{j}^{a, b}\right)^{(\alpha)}(t):={ }_{-1} D_{t}^{\alpha} J_{j}^{a, b}(t)=\gamma_{j, n}^{a, b} \hat{J}_{j-n}^{a+n, b+n, n-\alpha}(t), \quad n-1<\alpha<n,
$$

where $\gamma_{j, n}^{a, b}$ is a known constant.

Let $\left\{t_{i}\right\}_{i=0}^{N}$ be a set of collocation points, typically chosen as Gauss quadrature points. We define the fractional differentiation matrix of order $\alpha$ as

$$
\mathcal{D}_{i j}^{\alpha}=\left(J_{j}^{a, b}\right)^{(\alpha)}\left(t_{i}\right), \quad 0 \leqslant i, j \leqslant N .
$$

The entries of $\mathcal{D}^{\alpha}$ can easily be computed through (3.8)-(3.10).

We can recast this using a more convenient nodal representation of $u(t)$. Let $\left\{L_{j}^{i}(t)\right\}_{j=0}^{N}$ be Lagrange polynomials associated with $\left\{\xi_{j}^{i}\right\}_{j=0}^{N}$ where $\xi[-1,1]$ represents the affinely mapped time, $t$. We can then express $u(t)$ as

$$
u_{h}(t)=\sum_{l=0}^{N} u_{l}^{i} L_{l}^{i}(t)=\sum_{l=0}^{N} \hat{u}_{l}^{i} J_{l}^{a, b}(t), \quad t \in \Omega_{i} .
$$

If we assume that the expansion coefficients, $\hat{u}_{l}$, are computed through a quadrature over $\Omega_{i}$, we recover modal and nodal representations of $u_{h}(t)$ :

$$
\left(\hat{\boldsymbol{u}}^{i}\right)_{j}=\hat{u}_{j}^{i}, \quad\left(\boldsymbol{u}^{i}\right)_{j}=u_{h}\left(t_{j}^{i}\right), \quad 0 \leqslant j \leqslant N, \quad 1 \leqslant i \leqslant K
$$


connected through

$$
\mathcal{V} \hat{\boldsymbol{u}}^{i}=\boldsymbol{u}^{i}, l l \mathcal{V}_{i j}=J_{j}^{a, b}\left(t_{i}\right)
$$

where $\mathcal{V}$ is the Vandermonde matrix associated with the Legendre transformation (cf. [5]).

We can finally express the fractional derivative as

$$
{ }_{0} D_{t}^{-\alpha} u(t)=\sum_{l=0}^{N} \hat{u}_{l}\left(J_{j}^{a, b}\right)^{(\alpha)}(t),
$$

which, if evaluated the grid points, $t_{i}$, results in

$$
{ }_{0} D_{t}^{-\alpha} u(\mathbf{t})=\mathcal{D}^{\alpha} \hat{\boldsymbol{u}}=\mathcal{D}^{\alpha} \mathcal{V}^{-1} \boldsymbol{u},
$$

where we have dropped the index $i$ for simplicity.

\subsection{Spectral approximations in the multi-domain setting}

Let us now divide $\Omega=\left[t_{l}, t_{r}\right]$ into $K$ elements:

$$
t_{l}=t_{0}<t_{1}<\cdots<t_{K}=t_{r}, \quad \Omega_{i}=\left[t_{i-1}, t_{i}\right], \quad h_{i}=t_{i}-t_{i-1}, \quad 1 \leqslant i \leqslant K .
$$

For any $t \in \Omega_{i+1}$, we have $(n-1<\alpha<n)$ :

$$
\begin{aligned}
{ }_{0} D_{x}^{\alpha} u(t) & =\frac{1}{\Gamma(n-\alpha)} \int_{0}^{t}(t-s)^{n-1-\alpha} \frac{\mathrm{d}^{n} u(s)}{\mathrm{d} s^{n}} \mathrm{~d} s \\
& =\frac{1}{\Gamma(n-\alpha)} \sum_{k=1}^{i} \int_{\Omega_{k}}(t-s)^{n-1-\alpha} \frac{\mathrm{d}^{n} u(s)}{\mathrm{d} s^{n}} \mathrm{~d} s+\frac{1}{\Gamma(n-\alpha)} \int_{t_{i}}^{t}(t-s)^{n-1-\alpha} \frac{\mathrm{d}^{n} u(s)}{\mathrm{d} s^{n}} \mathrm{~d} s .
\end{aligned}
$$

The second part of this expression is, with appropriate scaling, equivalent to the one-domain problem discussed above. Hence, assuming that the solution locally is expressed as an expansion in Jacobi polynomials, the fractional derivative can be computed using 3.11.

Calculating the history part accurately is key to our multi-domain spectral method as this impacts both accuracy and computational cost of the method. We shall consider two different methods for achieving this and, subsequently, combine these to achieve a robust balance between cost and accuracy. In the following we refer to these two approaches as Differentiation-A and Differentiation-B and we shall illustrate shortly their individual benefits and challenges as motivation for considering a hybrid approach.

\subsubsection{Differentiation- $A$}

With a proper affine transformation of $\Omega_{k}$, we may consider the following quantity:

$$
\tilde{J}_{j}^{a, b, \alpha}(t):=\frac{1}{\Gamma(\alpha)} \int_{-1}^{1}(t-s)^{\alpha-1} J_{j}^{a, b}(s) \mathrm{d} s, \quad t>1, \quad 0 \leqslant j \leqslant N .
$$

Note that since we assume $t>1$ in the history part, the kernel is regular. 
Theorem 3.2. With this definition, $\left\{\tilde{J}_{j}^{a, b, \alpha}(t)\right\}_{j=0}^{N}$ satisfies the following three-term-recurrence relation:

$$
\begin{aligned}
& \tilde{J}_{0}^{a, b, \alpha}(t)=\frac{(t+1)^{\alpha}-(t-1)^{\alpha}}{\Gamma(\alpha+1)} \\
& \tilde{J}_{1}^{a, b, \alpha}(t)=\frac{a+b+2}{2 \Gamma(2+\alpha)}\left((t-\alpha)(t+1)^{\alpha}-(t+\alpha)(t-1)^{\alpha}\right)+\frac{a-b}{2} \tilde{J}_{0}^{a, b, \alpha}(t), \\
& \tilde{A}_{j}^{a, b} \tilde{J}_{j+1}^{a, b, \alpha}(t)=\tilde{B}_{j}^{a, b} \tilde{J}_{j}^{a, b, \alpha}(t)+\tilde{C}_{j}^{a, b} \tilde{J}_{j-1}^{a, b, \alpha}(t)+\tilde{D}_{j}^{a, b}(t+1)^{\alpha}+\tilde{E}_{j}^{a, b}(t-1)^{\alpha},
\end{aligned}
$$

where the coefficients are defined as

$$
\begin{aligned}
\tilde{A}_{j}^{a, b} & =\left(1+\alpha A_{j}^{a, b} \bar{C}_{j}^{a, b}\right), \\
\tilde{B}_{j}^{a, b} & =\left(A_{j}^{a, b} t-B_{j}^{a, b}-\alpha A_{j}^{a, b} \bar{B}_{j}^{a, b}\right), \\
\tilde{C}_{j}^{a, b} & =-\left(C_{j}^{a, b}+\alpha A_{j}^{a, b} \bar{A}_{j}^{a, b}\right), \\
\tilde{D}_{j}^{a, b} & =\frac{A_{j}^{a, b}}{\Gamma(\alpha)}\left(\bar{A}_{j}^{a, b} J_{j-1}^{a, b}(-1)+\bar{B}_{j}^{a, b} J_{j}^{a, b}(-1)+\bar{C}_{j}^{a, b} J_{j+1}^{a, b}(-1)\right), \\
\tilde{E}_{j}^{a, b} & =-\frac{A_{j}^{a, b}}{\Gamma(\alpha)}\left(\bar{A}_{j}^{a, b} J_{j-1}^{a, b}(1)+\bar{B}_{j}^{a, b} J_{j}^{a, b}(1)+\bar{C}_{j}^{a, b} J_{j+1}^{a, b}(1)\right) .
\end{aligned}
$$

Proof. For $J_{0}^{a, b}(t)$ and $J_{1}^{a, b}(t)$, one easily obtains

$$
\begin{aligned}
& \tilde{J}_{0}^{a, b, \alpha}(t)=\frac{1}{\Gamma(\alpha)} \int_{-1}^{1}(t-s)^{\alpha-1} \mathrm{~d} s=\frac{(t+1)^{\alpha}-(t-1)^{\alpha}}{\Gamma(\alpha+1)} \\
& \tilde{J}_{1}^{a, b, \alpha}(t)=\frac{a+b+2}{2 \Gamma(2+\alpha)}\left((t-\alpha)(t+1)^{\alpha}-(t+\alpha)(t-1)^{\alpha}\right)+\frac{a-b}{2} \tilde{J}_{0}^{a, b, \alpha}(t) .
\end{aligned}
$$

For $\left\{J_{j+1}^{a, b}(t), j \geqslant 1\right\}$, using the three terms recurrence relation (3.1), we recover

$$
\begin{aligned}
\tilde{J}_{j+1}^{a, b, \alpha}(t) & =\frac{1}{\Gamma(\alpha)} \int_{-1}^{1}(t-s)^{\alpha-1}\left(\left(A_{j}^{a, b} s-B_{j}^{a, b}\right) J_{j}^{a, b}(s)-C_{j}^{a, b} J_{j-1}^{a, b}(s)\right) \mathrm{d} s \\
& =-B_{j}^{a, b} \tilde{J}_{j}^{a, b, \alpha}(t)-C_{j}^{a, b} \tilde{J}_{j-1}^{a, b, \alpha}(t)+\frac{A_{j}^{a, b}}{\Gamma(\alpha)} \int_{-1}^{1}(t-s)^{\alpha-1} s J_{j}^{a, b}(s) \mathrm{d} s \\
& =-B_{j}^{a, b} \tilde{J}_{j}^{a, b, \alpha}(t)-C_{j}^{a, b} \tilde{J}_{j-1}^{a, b, \alpha}(t)-\frac{A_{j}^{a, b}}{\Gamma(\alpha)} \int_{-1}^{1}(t-s)^{\alpha-1}((t-s)-t) J_{j}^{a, b}(s) \mathrm{d} s \\
& =\left(x A_{j}^{a, b}-B_{j}^{a, b}\right) \tilde{J}_{j}^{a, b, \alpha}(t)-C_{j}^{a, b} \tilde{J}_{j-1}^{a, b, \alpha}(t)-\frac{A_{j}^{a, b}}{\Gamma(\alpha)} \int_{-1}^{1}(t-s)^{\alpha} J_{j}^{a, b}(s) \mathrm{d} s .
\end{aligned}
$$

Next, we deal with the last term in (3.25). Recalling (3.5), we can have

$$
\begin{aligned}
& \frac{1}{\Gamma(\alpha)} \int_{-1}^{1}(t-s)^{\alpha} J_{j}^{a, b}(s) \mathrm{d} s \\
& \quad=\frac{1}{\Gamma(\alpha)} \int_{-1}^{1}(t-s)^{\alpha}\left(\bar{A}_{j}^{a, b} \frac{\mathrm{d}}{\mathrm{d} s} J_{j-1}^{a, b}(s)+\bar{B}_{j}^{a, b} \frac{\mathrm{d}}{\mathrm{d} s} J_{j}^{a, b}(s)+\bar{C}_{j}^{a, b} \frac{\mathrm{d}}{\mathrm{d} s} J_{j+1}^{a, b}(s)\right) \mathrm{d} s .
\end{aligned}
$$


Through integration by parts, we obtain

$$
\begin{aligned}
& \frac{1}{\Gamma(\alpha)} \int_{-1}^{1}(t-s)^{\alpha} \bar{A}_{j}^{a, b} \frac{\mathrm{d}}{\mathrm{d} s} J_{j-1}^{a, b}(s) \mathrm{d} s=\frac{\bar{A}_{j}^{a, b}\left((t-1)^{\alpha} J_{j-1}^{a, b}(1)-(t+1)^{\alpha} J_{j-1}^{a, b}(-1)\right)}{\Gamma(\alpha)}-\alpha \bar{A}_{j}^{a, b} \tilde{J}_{j-1}^{a, b, \alpha}(t), \\
& \frac{1}{\Gamma(\alpha)} \int_{-1}^{1}(t-s)^{\alpha} \bar{B}_{j}^{a, b} \frac{\mathrm{d}}{\mathrm{d} s} J_{j}^{a, b}(s) \mathrm{d} s=\frac{\bar{B}_{j}^{a, b}\left((t-1)^{\alpha} J_{j}^{a, b}(1)-(t+1)^{\alpha} J_{j}^{a, b}(-1)\right)}{\Gamma(\alpha)}-\alpha \bar{B}_{j}^{a, b} \tilde{J}_{j}^{a, b, \alpha}(t) \\
& \frac{1}{\Gamma(\alpha)} \int_{-1}^{1}(t-s)^{\alpha} \bar{C}_{j}^{a, b} \frac{\mathrm{d}}{\mathrm{d} s} J_{j+1}^{a, b}(s) \mathrm{d} s=\frac{\bar{C}_{j}^{a, b}\left((t-1)^{\alpha} J_{j+1}^{a, b}(1)-(t+1)^{\alpha} J_{j+1}^{a, b}(-1)\right)}{\Gamma(\alpha)}-\alpha \bar{C}_{j}^{a, b} \tilde{J}_{j+1}^{a, b, \alpha}(t) .
\end{aligned}
$$

Combining (3.25)-(3.29), we obtain the three terms recurrence relation:

$$
\tilde{A}_{j}^{a, b} \tilde{J}_{j+1}^{a, b, \alpha}(t)=\tilde{B}_{j}^{a, b} \tilde{J}_{j}^{a, b, \alpha}(t)+\tilde{C}_{j}^{a, b} \tilde{J}_{j-1}^{a, b, \alpha}(t)+\tilde{D}_{j}^{a, b}(t+1)^{\alpha}+\tilde{E}_{j}^{a, b}(t-1)^{\alpha} .
$$

where $\tilde{A}_{j}^{a, b}, \tilde{B}_{j}^{a, b}, \tilde{C}_{j}^{a, b}, \tilde{D}_{j}^{a, b}, \tilde{E}_{j}^{a, b}$ are defined in (3.19).

With (3.17), we can calculate the integral term in the history part as

$$
\tilde{D}^{\alpha} J_{j}^{a, b}(t):=\frac{1}{\Gamma(n-\alpha)} \int_{-1}^{1}(t-s)^{n-\alpha-1} \frac{\mathrm{d}^{n}}{\mathrm{~d} s^{n}} J_{j}^{a, b}(s) \mathrm{d} s=\gamma_{j, n}^{a, b} \tilde{J}_{j-n}^{a+n, b+n, n-\alpha}(t), \quad t>1 .
$$

To test the accuracy of differentiation based on this recurrence relation, we consider numerical differentiation of a function $u(t)=\sin (t)$. Figure 1 illustrates the error of the numerical differentiation of order $\alpha$ of $u(t)$, by using Differentiation-A. The following parameters were used:

$$
\Omega=[0,2 \pi], \quad \alpha=0.6, \quad N=16, \quad K=14,
$$

where the same number of $N$ is used in all elements. What is worth observing in Figure 1 is that large numerical errors appear as $t$ gets large, i.e., as the history increases.

To understand this, consider the proof of Theorem 3.2. From (3.23) to (3.24), we use $-s=(t-s)-t$, where $s \in[-1,1]$ and $t>1$. Note that after the affine transformation the right end of each element corresponds to $s=1$ and cancellation becomes significant as observed in near $2 \pi$ in Figure 1 . For $t \gg 1$, even small errors destroy the real value through the recurrence. This is a classic phenomenon for forward recurrences and to compute $\tilde{J}_{j}^{a, b, \alpha}(t)$ for large $t$, we need to consider an alternative. 


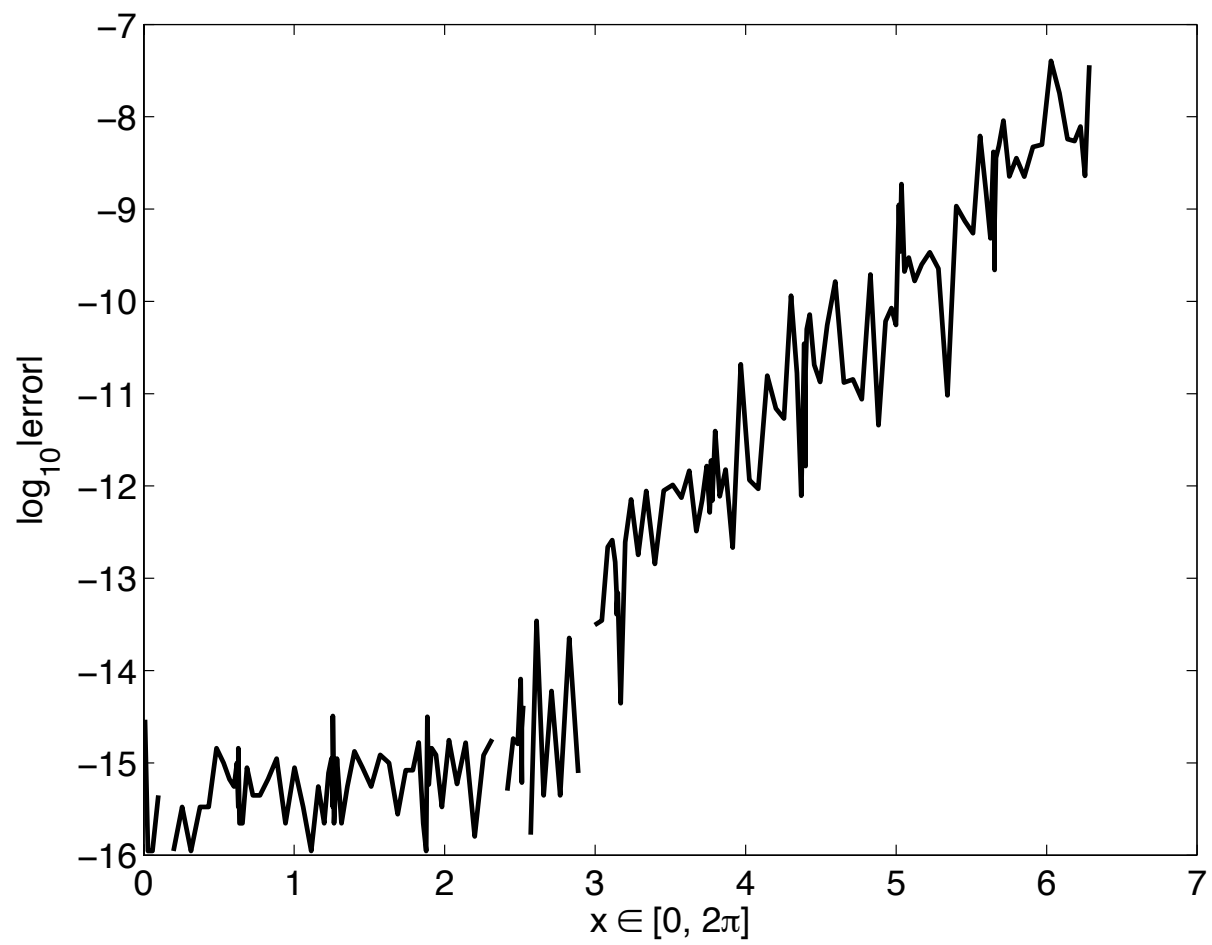

Figure 1: Numerical errors of Differentiation-A with $\sin (t)$ and parameters in (3.32).

\subsubsection{Differentiation- $B$}

As an alternative approach to calculate (3.17) we consider a Gauss quadrature

$$
\tilde{D}^{\alpha} J_{j}^{a, b}(t) \approx \frac{1}{\Gamma(n-\alpha)} \sum_{i=0}^{N} \omega_{i}\left(t-s_{i}\right)^{n-\alpha-1} \frac{\mathrm{d}^{n}}{\mathrm{~d} s^{n}} J_{j}^{a, b}\left(s_{i}\right),
$$

where $\left\{s_{i}, \omega_{i}\right\}$ can be chosen as the Legendre-Gauss-Lobatto points if $t$ is outside $[-1,1]$ since in this case the kernel is regular.

One easily observes that Differentiation-B can not be expected to be accurate when $(t-s)^{n-\alpha-1}$ is nearly singular, i.e., when $t$ is very near the boundary of $[-1,1]$. To illustrate this point, we consider the same example and parameters as in Section 3.2.1. Figure 2 highlights that there are indeed large errors near the right end of each element. They are caused by the singularity of $(t-s)^{n-\alpha-1}$. 


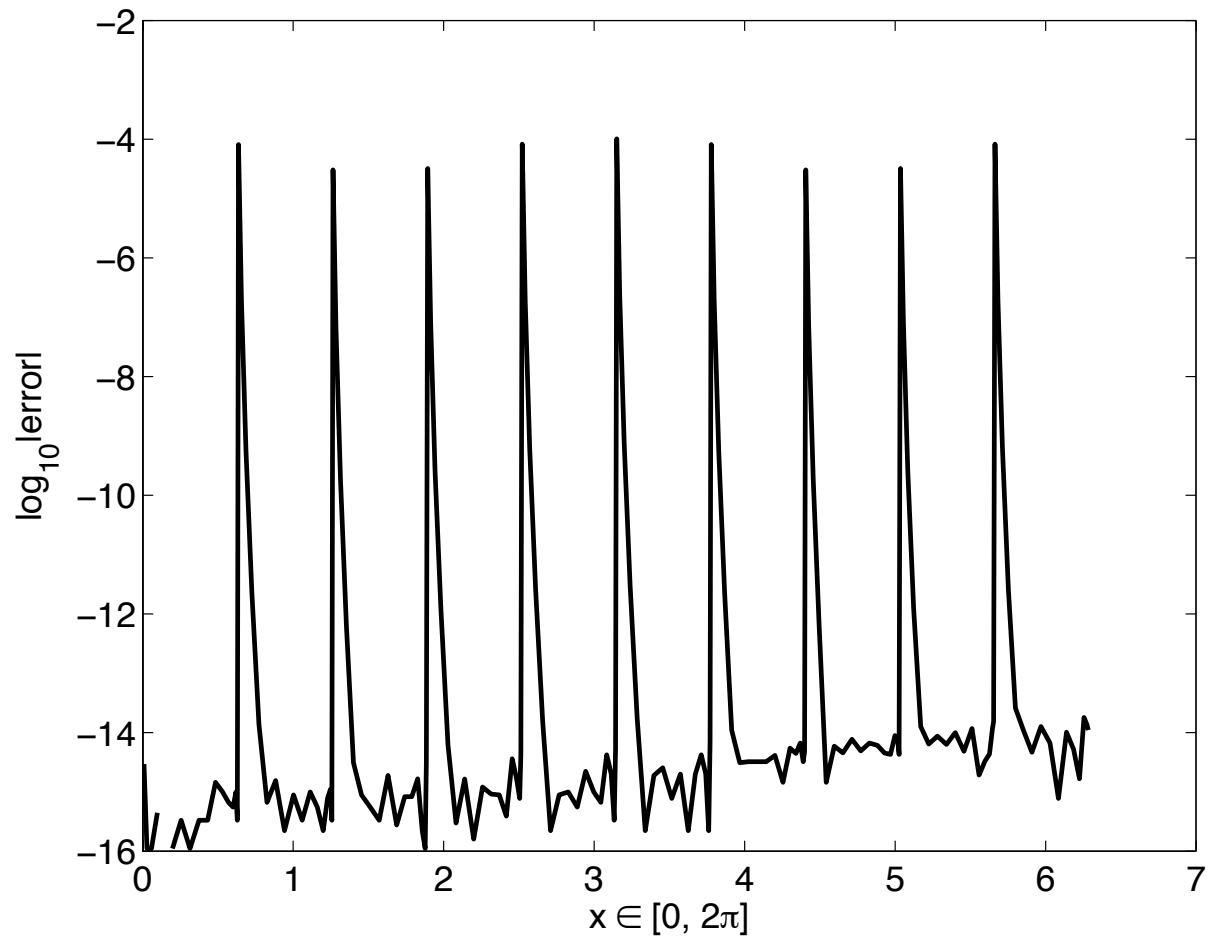

Figure 2: Numerical errors of Differentiation-B with $\sin (t)$ and parameters in (3.32).

\subsubsection{A Hybrid Approach}

Based on the discussion above, we can make the following observations. When $t$ is close to the end point of the integration domain, Differentiation-A performs well while Differentiation-B fails. As $t$ increases, the situation reverses, and Differentiation-B is superior to Differentiation-A.

This situation suggest that a hybrid of these two techniques has a potential for reaching high-order accuracy across the entire history by using Differentiation-A for $\Omega_{i-m}, \cdots, \Omega_{i}$, where $m$ is small integer, and use Differentiation-B for the remaining part of the history elements.

To illustrate the hybrid approach, we consider again the fractional differentiation of $\sin (t)$ and use the same parameters as in (3.32). We fix $m=3$, i.e., a total of four elements are computed with Differentiation$A$ and the rest are obtained with Differentiation-B. Figure 3 illustrates that the numerical errors in the hybrid approach are uniformly small across the entire range. 


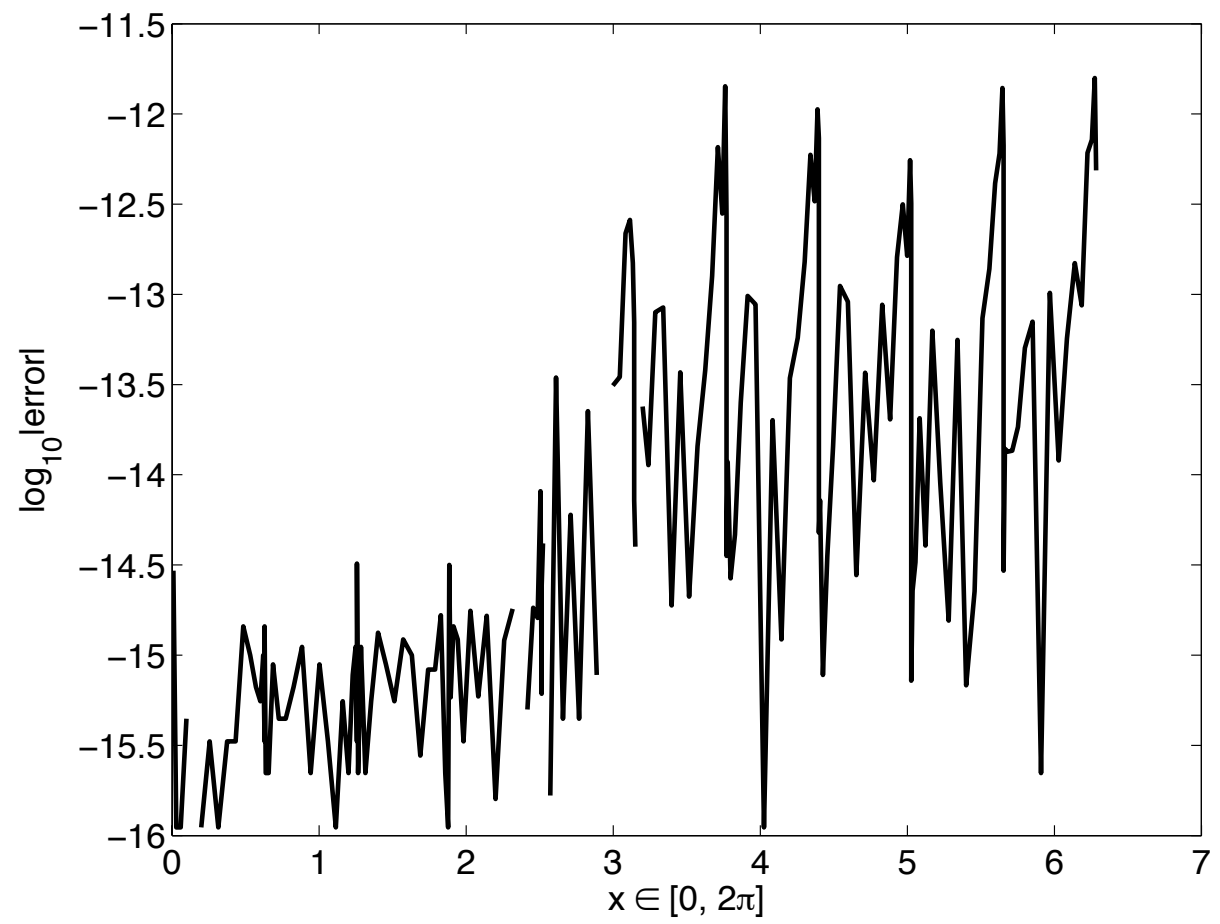

Figure 3: Numerical errors of the hybrid approach with $\sin (x)$ and parameters in (3.32).

\section{Multi-domain spectral method for TFDE}

Let us now return to the fractional differential equation

$$
\left\{\begin{array}{l}
{ }_{0} D_{t}^{\alpha} u(t)=\lambda u(t)+f(t), \quad t \in \Omega, \\
u(0)=u_{0}
\end{array}\right.
$$

where $\lambda \in \mathbb{C}$, and $\alpha \in(0,1)$. To understand the basic properties of our scheme, we shall first consider this model problem.

\subsection{Description of the Method}

Without loss of generality, we set $(a, b)=(0,0)$ for the index of the Jacobi polynomials, i.e., we use an expansion in Legendre polynomials to represent the solution.

First, consider a domain decomposition of $\Omega$ :

$$
0=t_{0}<t_{1}<\cdots<t_{K}=T, \quad \Omega_{i}=\left[t_{i-1}, t_{i}\right], \quad h_{i}=t_{i}-t_{i-1}, \quad 1 \leqslant i \leqslant K .
$$

The model equation (4.1) takes the form:

$$
\left\{\begin{array}{l}
t_{i-1} D_{t}^{\alpha} u(t)+\sum_{k=1}^{i-1} t_{k-1} \tilde{D}_{t_{k}}^{\alpha} u(t)=\lambda u(t)+f(t), \quad t \in \Omega_{i}, \quad 1 \leqslant i \leqslant K, \\
u(0)=u_{0},
\end{array}\right.
$$


where $\tilde{D}^{\alpha}$ is defined in (3.31).

Next, we define the approximating space:

$$
\mathbb{X}_{N}=\left\{u \in C(\Omega) ;\left.u\right|_{\Omega_{i}} \in \mathbb{P}_{N}, 1 \leqslant i \leqslant K\right\}
$$

where $\mathbb{P}_{N}$ is the polynomial space of degree $N$ defined on $\Omega_{i}$, i.e., we represent the global solution as piecewise polynomials.

For simplicity, we consider a uniform decomposition $\left(h_{i} \equiv h\right)$ and the same number of degrees of freedom on each element, although this is not required. The numerical solution can be expressed as

$$
u_{h}(t)=\sum_{l=0}^{N} \hat{u}_{l}^{i} P_{l}(\tilde{t}), \quad t \in \Omega_{i}
$$

where

$$
t=\frac{1-\tilde{t}}{2} t_{i-1}+\frac{1+\tilde{t}}{2} t_{i}, \quad \tilde{t} \in[-1,1]
$$

and $P_{l}(\tilde{t})$ is the $l$-th order Legendre polynomial defined on $[-1,1]$.

Insert (4.5) into (4.3), and enforce the equation to hold at a set of collocation points $\xi_{j}^{i}$, to recover

$$
{ }_{t_{i-1}} D_{t}^{\alpha} u_{h}\left(\xi_{j}^{i}\right)+\sum_{k=1}^{i-1} t_{k-1} \tilde{D}_{t_{k}}^{\alpha} u_{h}\left(\xi_{j}^{i}\right)=\lambda u_{h}\left(\xi_{j}^{i}\right)+f\left(\xi_{j}^{i}\right), \quad 0 \leqslant j \leqslant N, \quad 1 \leqslant i \leqslant K,
$$

We shall assume that $\left\{\xi_{j}^{i}\right\}_{j=0}^{N}$ is the Legendre-Gauss-Lobatto points on $\Omega_{i}$ but this too can be relaxed. Using the affine transformation in (4.6), we introduce the following matrices:

$$
\begin{aligned}
& \left(\hat{\mathcal{M}}_{0}\right)_{j l}=\left(\frac{h}{2}\right)_{-1}^{-\alpha} D_{t}^{\alpha} P_{l}\left(\xi_{j}\right), \quad 0 \leqslant j, l \leqslant N, \\
& \left(\hat{\mathcal{M}}_{i-k}\right)_{j l}=\left(\frac{h}{2}\right)_{-1}^{-\alpha} \tilde{D}_{1}^{\alpha} P_{l}\left(\tilde{\xi}_{j}^{i}\right), \quad 0 \leqslant j, l \leqslant N, \quad 1 \leqslant k \leqslant i-1,
\end{aligned}
$$

where

$$
\xi_{j}^{i}=\frac{1-\tilde{\xi}_{j}^{i}}{2} t_{k-1}+\frac{1+\tilde{\xi}_{j}^{i}}{2} t_{k}
$$

and $\tilde{D}^{\alpha}$ is defined in (3.31).

The multi-domain spectral method may now be expressed as follows. Assume that $\left\{\hat{\boldsymbol{u}}^{k}\right\}_{k=1}^{i-1}$ were solved on previous elements, i.e., the history is known. Then (4.7) is equivalent to the following linear system in $\hat{\boldsymbol{u}}^{i}$ :

$$
\hat{\mathcal{M}}_{0} \hat{\boldsymbol{u}}^{i}+\sum_{k=1}^{i-1} \hat{\mathcal{M}}_{i-k} \hat{\boldsymbol{u}}^{k}=\lambda \mathcal{V} \hat{\boldsymbol{u}}^{i}+\boldsymbol{f}^{i}
$$

where $\left(\boldsymbol{f}^{i}\right)_{j}=f\left(t_{j}^{i}\right)$. Following the discussion in Section 3, $\hat{\mathcal{M}}_{0}$ is calculated with the three-termrecurrence in Section 3.1.2, $\left\{\hat{\mathcal{M}}_{1}, \cdots, \hat{\mathcal{M}}_{m}\right\}$ are calculated with Differentiation-A, and $\left\{\hat{\mathcal{M}}_{m+1}, \cdots, \hat{\mathcal{M}}_{i-1}\right\}$ are calculated with Differentiation-B, where $m$ is a small integer like 3 or 4 . 
In practice, it is preferred to work directly in physical space. Hence, we write (4.10) as

$$
\mathcal{N}_{0} \boldsymbol{u}^{i}+\sum_{k=1}^{i-1} \mathcal{N}_{i-k} \boldsymbol{u}^{k}=\lambda\left(\frac{h}{2}\right)^{\alpha} \boldsymbol{u}^{i}+\left(\frac{h}{2}\right)^{\alpha} \boldsymbol{f}^{i}
$$

where

$$
\mathcal{N}_{k}=\left(\frac{h}{2}\right)^{\alpha} \hat{\mathcal{M}}_{k} \mathcal{V}^{-1}, \quad 0 \leqslant k \leqslant i-1
$$

To complete the method, one needs to enforce the initial condition on each element: the first row of (4.11) is replaced by $(1,0, \cdots, 0)$ and the first element of the right hand side vector is replaced by $u_{h}\left(t_{N}^{i-1}\right)$. If $\alpha \in(1,2)$, the second row of (4.11) also needs to be replaced by the first row of the first-order differentiation matrix, and the right hand side vector is changed accordingly.

\subsection{Stability}

Let us consider the stability characteristics of the proposed method. We consider the model equation:

$$
\left\{\begin{array}{l}
{ }_{0} D_{t}^{\alpha} u(t)=\lambda u(t), \quad t \in \Omega, \\
u(0)=u_{0} .
\end{array}\right.
$$

It is stated in Theorem 2.17 of [18] that (4.13) is stable if the following condition is satisfied

$$
|\arg (\lambda)|>\frac{\alpha \pi}{2}
$$

Following (4.11), the multi-domain spectral method is expressed through the following linear system

$$
\mathcal{N}_{0} \boldsymbol{u}^{i}+\sum_{k=1}^{i-1} \mathcal{N}_{i-k} \boldsymbol{u}^{k}=\lambda\left(\frac{h}{2}\right)^{\alpha} \boldsymbol{u}^{i},
$$

which can be recognized as a general linear method [4], albeit with a unbounded number of terms as the time step approaches zero. To study the stability of (4.15), we fix the number of terms in the summation, effectively eliminating the memory effect as it fades out. Denote the number of remaining terms as $N_{c}$ and consider

$$
\boldsymbol{u}^{i}-\sum_{k=1}^{N_{c}} \mathcal{R}_{i-k} \boldsymbol{u}^{k}=\mathbf{0}
$$

where

$$
\mathcal{R}_{k}=-\left(\mathcal{N}_{0}-\lambda\left(\frac{h}{2}\right)^{\alpha} \mathcal{I}\right)^{-1} \mathcal{N}_{k}
$$

Introduce the vector

$$
\boldsymbol{y}^{i}=\left(\boldsymbol{u}^{i}, \boldsymbol{u}^{i-1}, \cdots, \boldsymbol{u}^{2}\right)^{T}
$$

Then, (4.16) is equivalent to

$$
\boldsymbol{y}^{i}=\mathcal{P} \boldsymbol{y}^{i-1},
$$


where $\mathcal{P}$ is assembled from $\mathcal{R}_{k}$ as

$$
\mathcal{P}=\left(\begin{array}{cccc}
\mathcal{R}_{1} & \mathcal{R}_{2} & \mathcal{R}_{3} & \cdots \\
\mathcal{I} & & & \\
& \mathcal{I} & & \\
& & \mathcal{I} & \\
& \cdots & \cdots &
\end{array}\right)
$$

The stability of (4.15) is then determined by the spectrum of $\mathcal{P}$ as a function of $z=\lambda\left(\frac{h}{2}\right)^{\alpha}$.

In Figure 4, we plot the stability regions, defined as the regions where all eigenvalues have modulus less than one, for the linear case, i.e., $N=1$, for different values of $\alpha$ 's and $N_{c}$ 's. The $x$-axis ( $y$-axis) is the real (imaginary) part of $z$ and the black regions represent unstable regions. First of all we observe that as $N_{c}$ increases, the stability regions appears to converge. Typically, with $N_{c} \geqslant 15$ we obtain a reliable stability region for the scheme. Secondly, as $\alpha$ approaches 1 , the stability region of the scheme approaches that of the backward Euler scheme as one would expect. To consider the extension of this study to higher order methods, we consider the cases of $N=2,3,4,5$ in Figure 5. The conclusions follow that of the $N=1$ case. The results supports a conjecture that the scheme is A-stable for all orders.

Furthermore, we observe in Figure 5 that stability regions of the numerical method contain the stability region of the continuous equation. Specifically, the angle of the draw line from $(0,0)$ to first time this line touches the region of instability is independent of $N$ and about $\pi \alpha / 2$. It is consistent with the theoretical results in (4.14).

\section{Numerical examples}

In the following we consider various examples to illustrate the accuracy and efficiency of the proposed scheme. For this, we consider both fractional ordinary and partial differential equations.

\subsection{Fractional differential equations}

Example 5.1. Let us first consider the order of convergence of the multi-domain spectral method applied to (4.1) with $\alpha \in(0,1)$ and $\lambda=-1$. We choose the exact solution to be $u(t)=\sin (t)+t$, yielding the source function,

$$
f(t)=\frac{t^{1-\alpha}}{2}\left(E_{1,2-\alpha}(i t)+E_{1,2-\alpha}(-i t)\right)+\frac{t^{1-\alpha}}{\Gamma(2-\alpha)}+\sin (t)+t, \quad t \in(0,4 \pi],
$$

where $i=\sqrt{-1}$ and $E_{\lambda, \mu}(\cdot)$ is the Mittag-Leffler function [1]

For the numerical results, let $K_{1}, K_{2}$ be the number of elements of two different meshes, and $e_{h, 1}, e_{h, 2}$ be the corresponding errors. Then, the order of convergence of the scheme is obtained as

$$
\text { Order }=\frac{\log \left(\left\|e_{h, 1}\right\|_{2} /\left\|e_{h, 2}\right\|_{2}\right)}{\log \left(K_{2} / K_{1}\right)}
$$

Numerical results for different values of $N, K$, and $\alpha$ are shown in Table 1 , showing $h p$-convergence as

$$
\left\|u-u_{h}\right\|_{2} \sim h^{N+1-\alpha} .
$$



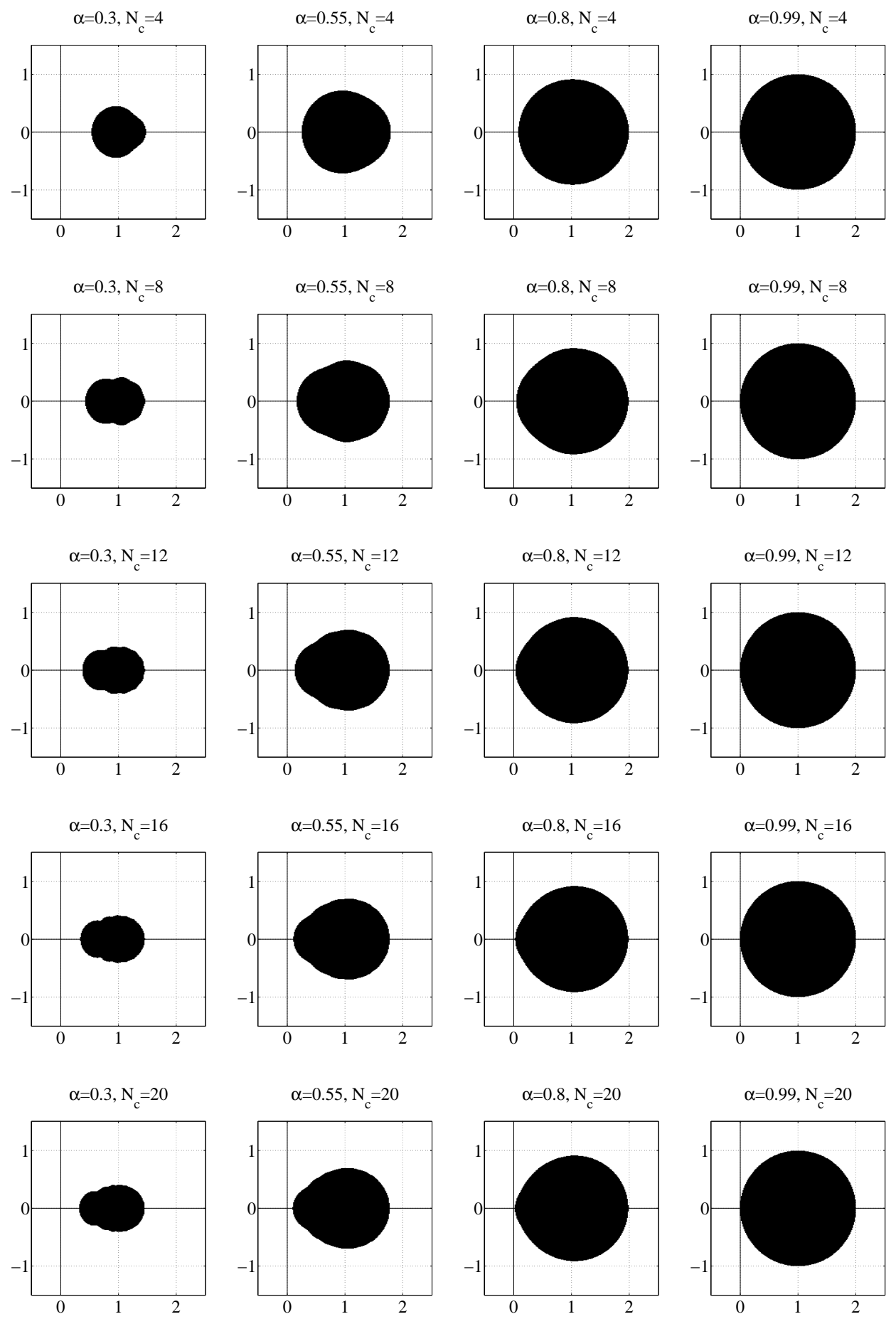

Figure 4: Stability domains for the multi-domain spectral method with $N=1$ (linear approximation). $N_{c}$ is the number of cut-off in the history for different values of $\alpha$. 

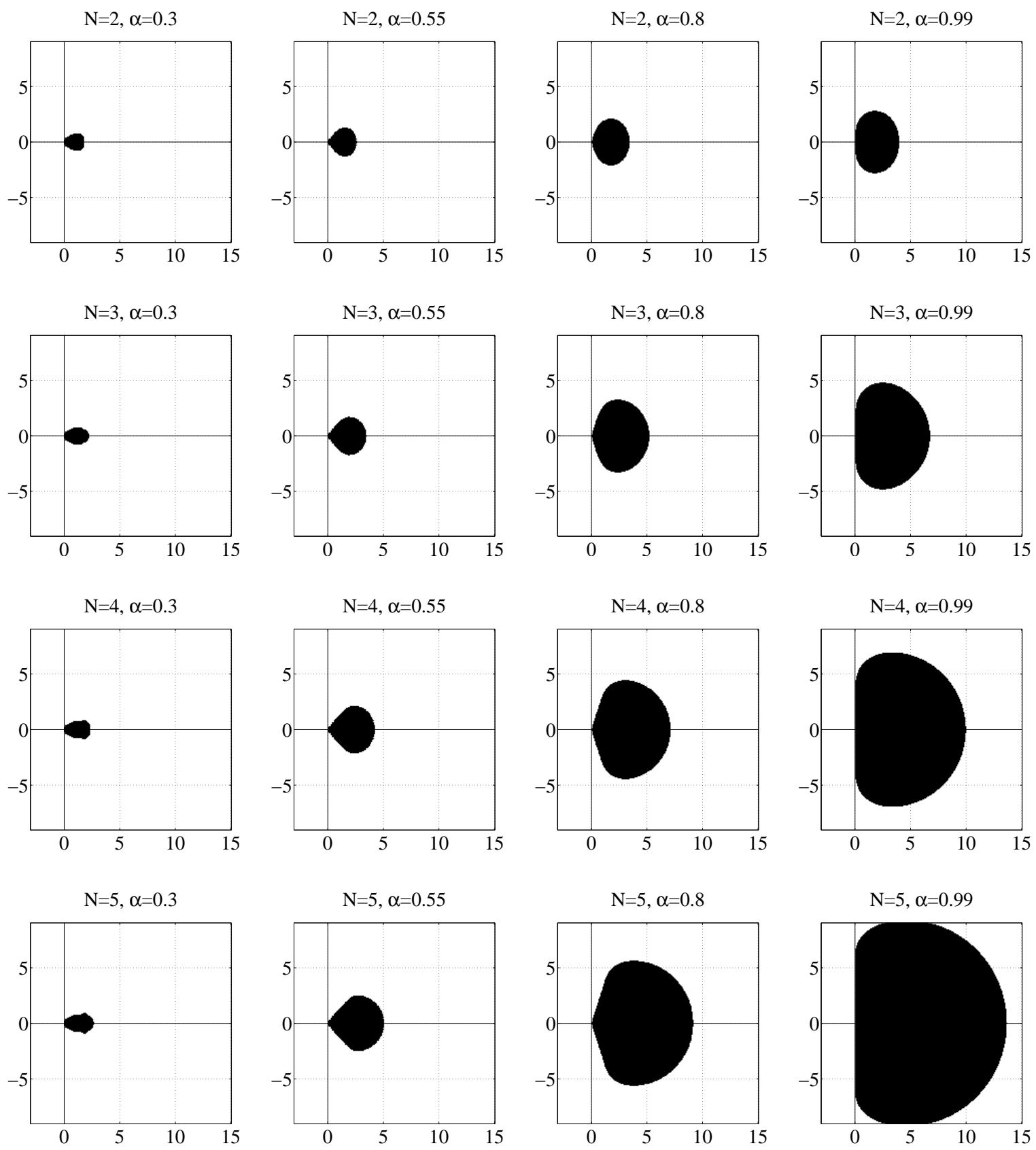

Figure 5: Numerical stability for the multi-domain spectral method with different $N$ and $\alpha$ values. $N_{c}$ is fixed as 20 . 


\begin{tabular}{|c|c|c|c|c|c|c|}
\hline \multirow[b]{3}{*}{$K$} & \multicolumn{6}{|c|}{$N=1$} \\
\hline & \multicolumn{2}{|c|}{$\alpha=0.1$} & \multicolumn{2}{|c|}{$\alpha=0.5$} & \multicolumn{2}{|c|}{$\alpha=0.9$} \\
\hline & $\left\|e_{h}\right\|_{2}$ & Order & $\left\|e_{h}\right\|_{2}$ & Order & $\left\|e_{h}\right\|_{2}$ & Order \\
\hline 20 & $\bar{~} 1.56 \mathrm{e}-02$ & 1.67 & $21.29 \mathrm{e}-01$ & 1.38 & $21.56 \mathrm{e}-02$ & 1.01 \\
\hline 30 & e- 03 & 1.6 & 10e- 02 & 1. & 91e-03 & 1.03 \\
\hline 40 & $4 e-03$ & 1.62 & $96 \mathrm{e}-02$ & 1.39 & $4.94 \mathrm{e}-03$ & 1.04 \\
\hline \multirow[t]{2}{*}{50} & $.44 \mathrm{e}-03$ & - & $3.64 \mathrm{e}-02$ & - & $1.55 \mathrm{e}-01$ & - \\
\hline & \multicolumn{6}{|c|}{$\bar{N} N=2$} \\
\hline & \multicolumn{2}{|c|}{$\alpha=0.1$} & \multicolumn{2}{|c|}{$\alpha=0.5$} & \multicolumn{2}{|c|}{$\alpha=0.9$} \\
\hline$K$ & $\left\|e_{h}\right\|_{2}$ & Order & $\left\|e_{h}\right\|_{2}$ & Order & $\left\|e_{h}\right\|_{2}$ & Order \\
\hline 20 & $.54 \mathrm{e}-04$ & 97 & $.62 \mathrm{e}-03$ & 2.78 & $4.54 \mathrm{e}-04$ & 2.15 \\
\hline 30 & e- & 2.9 & 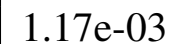 & 2.18 & $36 e-04$ & 2.11 \\
\hline 40 & e-05 & 2.97 & 30e-04 & 2.73 & $5.79 \mathrm{e}-05$ & 2.10 \\
\hline \multirow[t]{3}{*}{50} & 8e-05 & - & $2.88 \mathrm{e}-04$ & 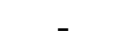 & $2.34 \mathrm{e}-03$ & - \\
\hline & \multicolumn{6}{|c|}{$N=3$} \\
\hline & \multicolumn{2}{|c|}{$\alpha=0.1$} & \multicolumn{2}{|c|}{$\alpha=0.5$} & \multicolumn{2}{|c|}{$\alpha=0.9$} \\
\hline$K$ & $\left\|e_{h}\right\|_{2}$ & Order & $\left\|e_{h}\right\|_{2}$ & Order & $\left\|e_{h}\right\|_{2}$ & Order \\
\hline 20 & $.76 \mathrm{e}-05$ & 3.94 & $.51 \mathrm{e}-04$ & 80 & $1.76 e-05$ & 3.20 \\
\hline 30 & $6 e-06$ & 3.95 & $24 \mathrm{e}-05$ & 3.80 & $.56 \mathrm{e}-06$ & 3.15 \\
\hline 40 & $4 e-06$ & 3.95 & 08e-05 & 3.80 & $1.14 \mathrm{e}-06$ & 3.12 \\
\hline \multirow[t]{3}{*}{50} & 74e-07 & - & $4.65 e-06$ & - & $3.96 e-05$ & - \\
\hline & \multicolumn{6}{|c|}{$N=4$} \\
\hline & \multicolumn{2}{|c|}{$\alpha=0.1$} & \multicolumn{2}{|c|}{$\alpha=0.5$} & \multicolumn{2}{|c|}{$\alpha=0.9$} \\
\hline$K$ & $\left\|e_{h}\right\|_{2}$ & Order & $\left\|e_{h}\right\|_{2}$ & Order & $\left\|e_{h}\right\|_{2}$ & Order \\
\hline 20 & $5 e-07$ & 96 & $11 \mathrm{e}-06$ & 4.86 & $5.05 e-07$ & 4.23 \\
\hline 30 & 7e-08 & 4.96 & $2 e-07$ & 4.86 & $6.77 e-08$ & 4.17 \\
\hline 40 & $63 e-08$ & 4.96 & $1.41 \mathrm{e}-07$ & 4.86 & $1.63 \mathrm{e}-08$ & 4.14 \\
\hline \multirow[t]{3}{*}{50} & 38e-09 & - & $4.77 \mathrm{e}-08$ & - & $4.57 e-07$ & - \\
\hline & \multicolumn{6}{|c|}{$N=5$} \\
\hline & \multicolumn{2}{|c|}{$\alpha=0.1$} & \multicolumn{2}{|c|}{$\alpha=0.5$} & \multicolumn{2}{|c|}{$\alpha=0.9$} \\
\hline$K$ & $\left\|e_{h}\right\|_{2}$ & Order & $\left\|e_{h}\right\|_{2}$ & Order & $\left\|e_{h}\right\|_{2}$ & Order \\
\hline 20 & $3 e-08$ & 5.9 & $.09 \mathrm{e}-07$ & 5.8 & $1.33 \mathrm{e}-08$ & 5.21 \\
\hline 30 & e-09 & 5.80 & -08 & 5.8 & $1.19 \mathrm{e}-09$ & 5.15 \\
\hline 40 & e- 10 & - & $1.85 \mathrm{e}-09$ & 5.89 & $2.24 \mathrm{e}-10$ & 5.13 \\
\hline 50 & $8.70 \mathrm{e}-11$ & - & $4.98 \mathrm{e}-10$ & & $5.23 e-09$ & \\
\hline
\end{tabular}

Table 1: Numerical errors and orders of convergence for Example 5.1 for number of elements $K$ and order of approximation $N$. 
Example 5.2. Next, we consider cases where $\alpha \in(1,2)$ and choose the exact solution to be $u(t)=$ $\sin \left(\frac{t}{2}\right)+2 e^{-\frac{t}{3}}$, resulting in a source function,

$$
\left.f(t)=\frac{i t^{2-\alpha}}{8}\left(E_{1,3-\alpha}\left(\frac{1}{2} i t\right)-E_{1,3-\alpha}\left(-\frac{1}{2} i t\right)\right)\right)+\frac{2 t^{2-\alpha}}{9} E_{1,3-\alpha}\left(-\frac{t}{3}\right)+\sin \left(\frac{t}{2}\right)+2 e^{-\frac{t}{3}}, \quad t \in(0,8 \pi] .
$$

Numerical results are shown in Table 2, displaying order of convergence as in (5.2).

Example 5.3. Let us now consider a time fractional PDE of the form:

$$
\begin{cases}{ }_{0} D_{t}^{\alpha} u+b_{0} D_{t}^{2 \alpha} u=c u_{x}+d u_{x x}+f, & (x, t) \in \Omega^{x} \times \Omega^{t}=(0,2 \pi) \times(0, T], \\ u(x, 0)=\phi(x), & x \in \Omega^{x}, \\ u_{t}(x, 0)=\psi(x), & x \in \Omega^{x},\end{cases}
$$

where $u=u(x, t)$ satisfies a $2 \pi$-periodic boundary condition and $1 / 2<\alpha<1$. If $b=0$, the initial velocity $u_{t}(x, 0)=\psi(x)$ is not needed.

Since $u$ and $f$ are periodic in the spatial domain, they are expressed as

$$
u_{h}(x, t)=\sum_{|k| \leq M / 2} \hat{u}_{k}(t) e^{i k x}, \quad f_{h}(x, t)=\sum_{|k| \leq M / 2} \hat{f}_{k}(t) e^{i k x}
$$

where $i=\sqrt{-1}$ and $\hat{f}_{k}(t)$ can be obtained through the fast Fourier transform (FFT).

Inserting (5.4) into (5.3) and requiring the equation to be satisfies in a Fourier Galerkin sense [9] yields a multi-term time-fractional differential equations:

$$
\forall k:{ }_{0} D_{t}^{\alpha} \hat{u}_{k}(t)+b_{0} D_{t}^{2 \alpha} \hat{u}_{k}(t)=i c k \hat{u}_{k}(t)-d k^{2} \hat{u}_{k}(t)+\hat{f}_{k}(t), \quad k \in \boldsymbol{I},
$$

for which we apply the multi-domain spectral method in the temporal direction. Once $\hat{u}_{k}$ 's are recovered, $u_{h}$ are obtained through the inverse fast Fourier transform.

We first consider $(5.3)$ in $(0,2 \pi) \times(0,10]$ with $b=1, c=0, d=1$, known as the fractional telegraph equation. The exact solution is taken to be $u(x, t)=\exp (\sin (x)) \sin (t)$, yielding the initial position $\phi(x)=0$, the initial velocity $\psi(x)=\exp (\sin (x))$ and the source term as

$$
f(x, t)=\exp (\sin (x))\left(\sin (x)-(\cos (x))^{2}\right) \sin (t)+\exp (\sin (x))\left(\sin ^{(\alpha)}(t)+\sin ^{(2 \alpha)}(t)\right),
$$

where $\sin ^{(\beta)}(t)=-\frac{1}{2} i^{n+1} t^{n-\beta}\left(E_{1, n-\beta+1}(i t)-(-1)^{n} E_{1, n-\beta+1}(-i t)\right)$ and $n=\lceil\beta\rceil$. We take $\alpha$ as 0.7 and $M$ as 40 and consider cases with $N=2,5,8,11$ and $K=4,6,8,10$. The numerical orders of convergence are shown in Figure 6. We observe $h p$-convergence in time as expected since the spatial error is negligible. 


\begin{tabular}{|c|c|c|c|c|c|c|}
\hline \multirow[b]{3}{*}{$K$} & \multicolumn{6}{|c|}{$N=$} \\
\hline & \multicolumn{2}{|c|}{$\alpha=1.1$} & \multicolumn{2}{|c|}{$\alpha=1.4$} & \multicolumn{2}{|c|}{$\alpha=1.8$} \\
\hline & $\left\|e_{h}\right\|_{2}$ & Order & $\left\|e_{h}\right\|_{2}$ & Order & $\left\|e_{h}\right\|_{2}$ & Order \\
\hline 20 & $.95 \mathrm{e}-02$ & 1.91 & $21.45 \mathrm{e}-01$ & 1.57 & $26.95 e-02$ & 1.11 \\
\hline 30 & e- 02 & 1.9 & $56 e-02$ & 1. & $20 \mathrm{e}-02$ & 1.13 \\
\hline 40 & $5 e-02$ & 1.90 & $86 \mathrm{e}-02$ & 1.58 & $.85 \mathrm{e}-02$ & 1.14 \\
\hline \multirow[t]{2}{*}{50} & $21 \mathrm{e}-02$ & - & $3.42 \mathrm{e}-02$ & - & $1.07 \mathrm{e}-01$ & - \\
\hline & \multicolumn{6}{|c|}{$N=3$} \\
\hline & \multicolumn{2}{|c|}{$\alpha=1.1$} & \multicolumn{2}{|c|}{$\alpha=1.4$} & \multicolumn{2}{|c|}{$\alpha=1.8$} \\
\hline K & $\left\|e_{h}\right\|_{2}$ & Order & $\left\|e_{h}\right\|_{2}$ & Order & $\left\|e_{h}\right\|_{2}$ & Order \\
\hline 20 & $.22 \mathrm{e}-03$ & 2.84 & $7.79 \mathrm{e}-03$ & 2.46 & $3.22 \mathrm{e}-03$ & 2.02 \\
\hline 30 & $e-03$ & 2.8 & $7 e-03$ & 2.51 & $.02 \mathrm{e}-03$ & 2.10 \\
\hline 40 & e-04 & 2.86 & $1.39 \mathrm{e}-03$ & 2.54 & $4.49 \mathrm{e}-04$ & 2.14 \\
\hline \multirow[t]{3}{*}{50} & 7e-04 & - & 7.91e-04 & - & $3.70 \mathrm{e}-03$ & - \\
\hline & \multicolumn{6}{|c|}{$\bar{N} N=4$} \\
\hline & \multicolumn{2}{|c|}{$\alpha=1.1$} & \multicolumn{2}{|c|}{$\alpha=1.4$} & \multicolumn{2}{|c|}{$\alpha=1.8$} \\
\hline$K$ & $\left\|e_{h}\right\|_{2}$ & Order & $\left\|e_{h}\right\|_{2}$ & Order & $\left\|e_{h}\right\|_{2}$ & Order \\
\hline 20 & $13 \mathrm{e}-04$ & 3.87 & $3.58 \mathrm{e}-04$ & 3.52 & $1.13 e-04$ & 3.18 \\
\hline 30 & $5 e-05$ & 3.87 & $77 \mathrm{e}-05$ & 3.55 & $.35 \mathrm{e}-05$ & 3.20 \\
\hline 40 & $4 e-06$ & 3.87 & $08 \mathrm{e}-05$ & 3.57 & $7.74 \mathrm{e}-06$ & 3.20 \\
\hline \multirow[t]{2}{*}{50} & $26 \mathrm{e}-06$ & - & $1.39 \mathrm{e}-05$ & - & $9.28 \mathrm{e}-05$ & 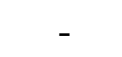 \\
\hline & \multicolumn{6}{|c|}{$N=5$} \\
\hline & \multicolumn{2}{|c|}{$\alpha=1.1$} & \multicolumn{2}{|c|}{$\alpha=1.4$} & \multicolumn{2}{|c|}{$\alpha=1.8$} \\
\hline K & $\left\|e_{h}\right\|_{2}$ & Order & $\left\|e_{h}\right\|_{2}$ & Order & $\left\|e_{h}\right\|_{2}$ & Order \\
\hline 20 & $5 e-06$ & 4.87 & $.34 \mathrm{e}-05$ & 55 & $3.55 \mathrm{e}-06$ & 4.19 \\
\hline 30 & $2 \mathrm{e}-07$ & 4.87 & $2 e-06$ & 4.57 & $4.92 \mathrm{e}-07$ & 4.19 \\
\hline 40 & $21 \mathrm{e}-07$ & 4.87 & 5.70e-07 & 4.58 & $1.21 \mathrm{e}-07$ & 4.20 \\
\hline \multirow[t]{3}{*}{50} & $8 \mathrm{e}-08$ & - & $2.05 \mathrm{e}-07$ & 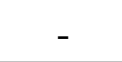 & $1.67 \mathrm{e}-06$ & 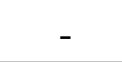 \\
\hline & \multicolumn{6}{|c|}{$N=6$} \\
\hline & \multicolumn{2}{|c|}{$\alpha=1.1$} & \multicolumn{2}{|c|}{$\alpha=1.4$} & \multicolumn{2}{|c|}{$\alpha=1.8$} \\
\hline$K$ & $\left\|e_{h}\right\|_{2}$ & Order & $\left\|e_{h}\right\|_{2}$ & Order & $\left\|e_{h}\right\|_{2}$ & Order \\
\hline 20 & $\overline{1 e-08}$ & & $1.08 \mathrm{e}-07$ & 5.5 & $9.11 \mathrm{e}-08$ & 5.22 \\
\hline 30 & & 5.8 & $3 e-08$ & 5.5 & $8.31 \mathrm{e}-09$ & 5.21 \\
\hline 40 & e-09 & 5.86 & $8.46 \mathrm{e}-09$ & 5.61 & $1.53 e-09$ & 5.21 \\
\hline 50 & $4.13 \mathrm{e}-10$ & & $2.42 \mathrm{e}-09$ & & $2.56 e-08$ & \\
\hline
\end{tabular}

Table 2: Numerical errors and orders of convergence for Example 5.2 for number of elements $K$ and order of approximation $N$. 


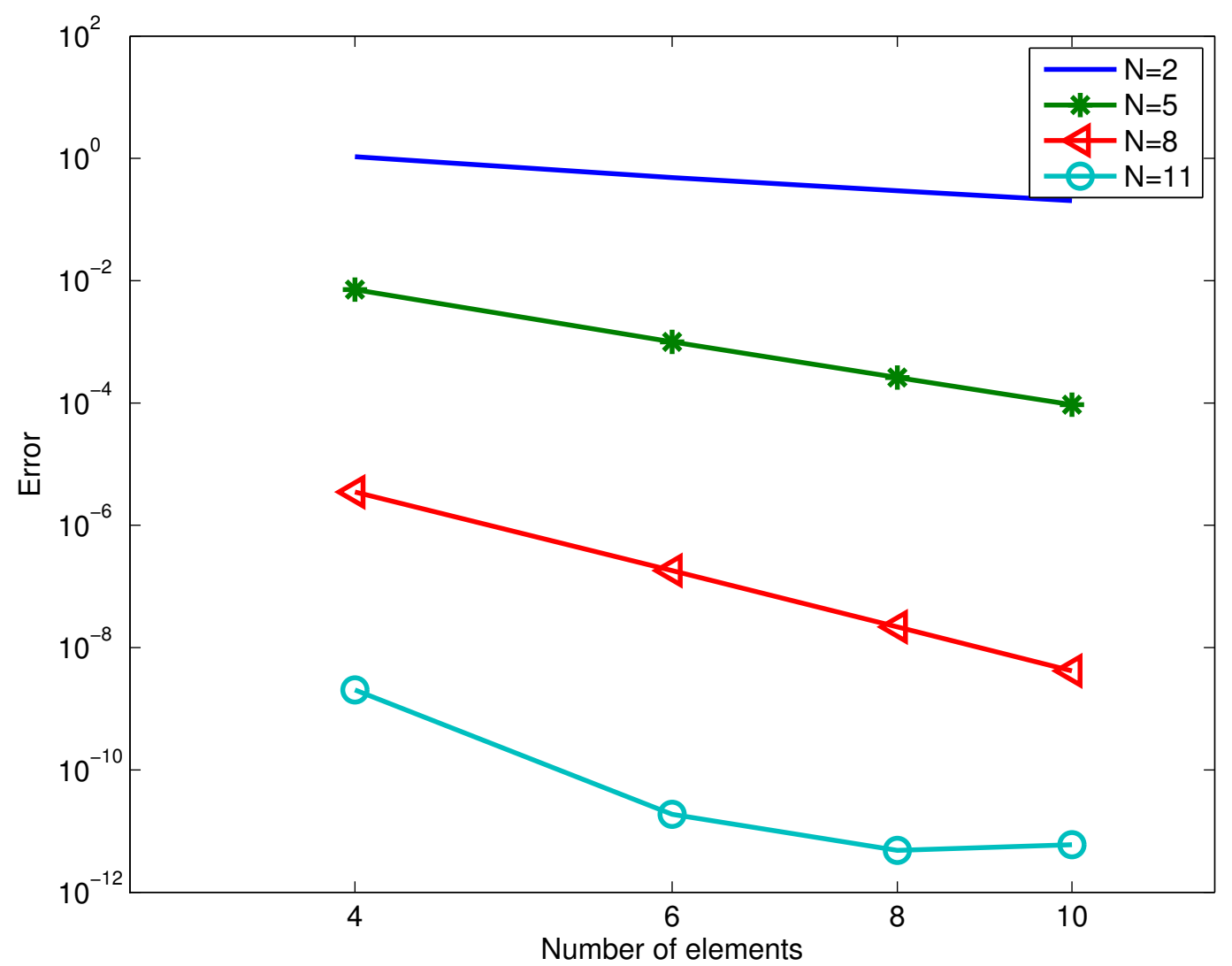

Figure 6: Convergence tests of equation (5.3) with different values of $N$ and $K$. The errors are measured in the $L_{2}$-norm.

Example 5.4. We finally consider the time-fractional convection diffusion equation, for which $b=0, f=$ 0 in (5.3):

$$
{ }_{0} D_{t}^{\alpha} u=c u_{x}+d u_{x x}, \quad(x, t) \in(0,10) \times(0,8],
$$

with the initial condition $u(x, 0)=\exp \left(-20(x-3)^{2}\right)$ and periodic boundary conditions. The initial condition is not strictly periodic, but it is so close to 0 at the two ends of the domain that it can be regarded as periodic in practice.

We use the following parameters:

$$
c=\frac{1}{5}, d=\frac{1}{100}, \alpha=0.2,0.5,0.8,1.0, N=3, K=30, M=200 .
$$

In Figure 7, we can observe that the advection effect becomes stronger as $\alpha$ becomes bigger.

\section{Conclusion}

We have proposed a new multi-domain spectral method for high-order integrations of the timefractional differential equation. To ensure a proper balance between computational cost and high-order accuracy, we have proposed a hybrid approach which combines two integration/differentiation rules, 


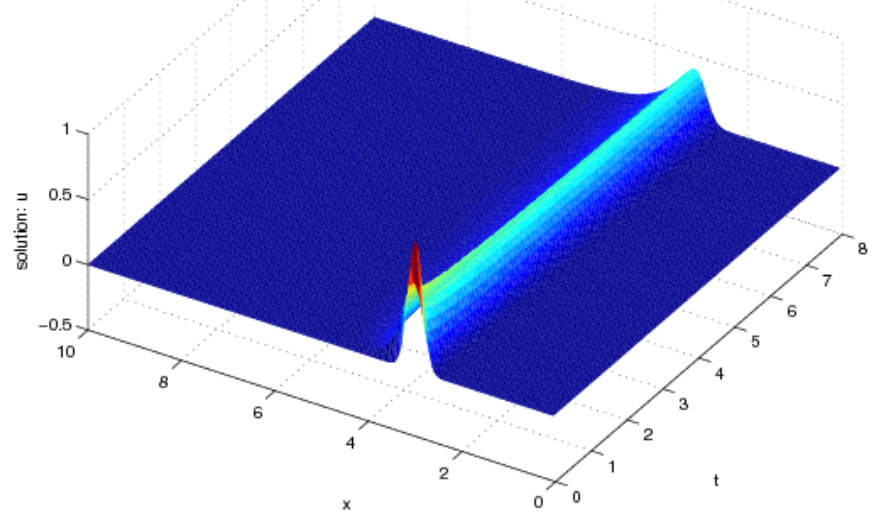

(a) $\alpha=0.2$

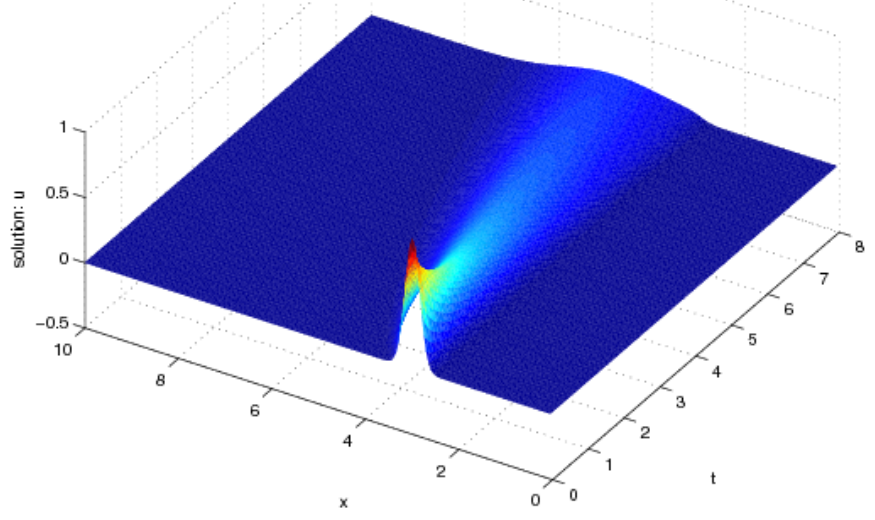

(c) $\alpha=0.8$

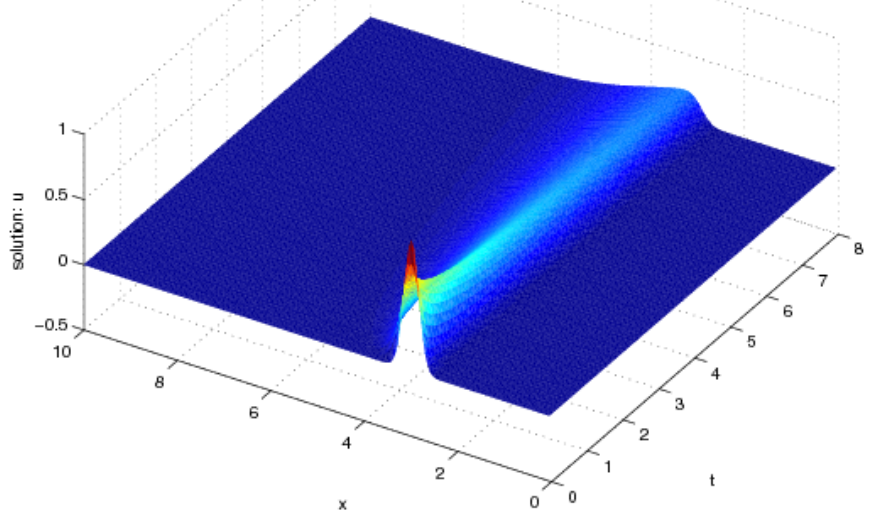

(b) $\alpha=0.5$

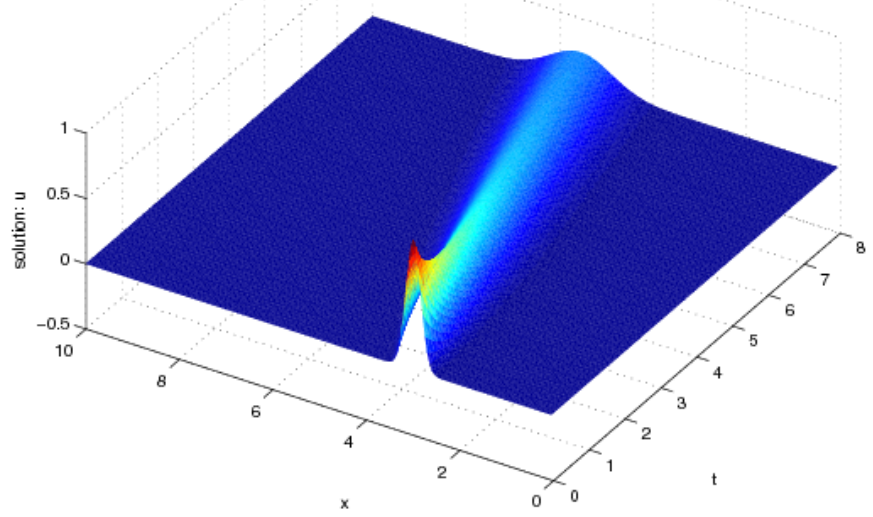

(d) $\alpha=1.0$

Figure 7: Numerical results for the time-fractional convection diffusion equation in Example 5.4. 
based on a the three-term-recurrence relation and Gauss quadrature, respectively. The stability analysis confirms excellent stability properties and the numerical results shows that the proposed method achieves $h p$-convergence for both TFDEs and TFPDEs.

\section{Acknowledgement}

The first and the third authors are partially support by OSD/AFOSR FA9550-09-1-0613 and AFOSR FA9550-12-1-0463. The second author was supported by the Hunan Provincial Innovation Foundation for Postgraduates (No. CX2011B080), the Excellent Doctoral Dissertation Foundation of Central South University and NSF DMS-1115416.

\section{References}

[1] M Abramowitz and I.A. Stegun. Handbook of Mathematical Functions. Dover Publishing, 1972.

[2] Ali H. Bhrawy and M. Alshomrani. A shifted legendre spectral method for fractional-order multipoint boundary value problems. Advan. Differ. Eqs, 8, 2012.

[3] Kevin Burrage, Nicholas Hale, and David Kay. An efficient implicit FEM scheme for fractional-inspace reaction-diffusion equations. SIAM Journal on Scientific Computing, 34(4):A2145-A2172, January 2012.

[4] J.C. Butcher. General linear methods. Acta Numerica, 15:157-256, 2006.

[5] Claudio Canuto, M. Yousuff Hussaini, Alfio Quarteroni, and Thomas A. Zang. Spectral Methods: Fundamentals in Single Domains. Springer, softcover reprint of hardcover 1st ed. 2006 edition, November 2010.

[6] V. B. L. Chaurasia and Devendra Kumar. Solution of the time-fractional navier-stokes equation. Gen, 4(2):4959, 2011.

[7] Moustafa El-Shahed and Ahmed Salem. On the generalized NavierStokes equations. Applied Mathematics and Computation, 156(1):287-293, August 2004.

[8] Boling Guo and Zhaohui Huo. Well-posedness for the nonlinear fractional schrdinger equation and inviscid limit behavior of solution for the fractional ginzburg-landau equation. Fractional Calculus and Applied Analysis, 16(1):226-242, April 2013.

[9] Jan S. Hesthaven, Sigal Gottlieb, and David Gottlieb. Spectral Methods for Time-Dependent Problems. Cambridge University Press, January 2007.

[10] Aditya Jaishankar and Gareth H. McKinley. Power-law rheology in the bulk and at the interface: quasi-properties and fractional constitutive equations. Proceedings of the Royal Society A: Mathematical, Physical and Engineering Science, 469(2149), January 2013.

[11] M. Khan, S. Hyder Ali, and Haitao Qi. On accelerated flows of a viscoelastic fluid with the fractional burgers model. Nonlinear Analysis: Real World Applications, 10(4):2286-2296, August 2009. 
[12] Changpin Li, Fanhai Zeng, and Fawang Liu. Spectral approximations to the fractional integral and derivative. Fractional Calculus and Applied Analysis, 15(3):383-406, June 2012.

[13] Xianjuan Li and Chuanju Xu. A space-time spectral method for the time fractional diffusion equation. SIAM Journal on Numerical Analysis, 47(3):2108-2131, January 2009.

[14] Yumin Lin and Chuanju Xu. Finite difference/spectral approximations for the time-fractional diffusion equation. Journal of Computational Physics, 225(2):1533-1552, August 2007.

[15] V.E. Lynch, B.A. Carreras, D. del Castillo-Negrete, K.M. Ferreira-Mejias, and H.R. Hicks. Numerical methods for the solution of partial differential equations of fractional order. Journal of Computational Physics, 192(2):406-421, December 2003.

[16] J. Tenreiro Machado, Alexandra M. Galhano, and Juan J. Trujillo. Science metrics on fractional calculus development since 1966. Fractional Calculus and Applied Analysis, 16(2):479-500, June 2013.

[17] J. Tenreiro Machado, Virginia Kiryakova, and Francesco Mainardi. Recent history of fractional calculus. Communications in Nonlinear Science and Numerical Simulations, 16:1140-1153, March 2011.

[18] Denis Matignon. Stability properties for generalized fractional differential systems. ESAIM: Proceedings, 5:145-158, 1998.

[19] Kassem Mustapha and William McLean. Superconvergence of a discontinuous galerkin method for fractional diffusion and wave equations. SIAM Journal on Numerical Analysis, 51(1):491-515, January 2013.

[20] Igor Podlubny. Fractional Differential Equations, Volume 198: An Introduction to Fractional Derivatives, Fractional Differential Equations, to Methods of Their ... Academic Press, 1 edition, November 1998.

[21] Gabor Szegö. Orthongonal Polynomials. American Mathematical Soc., July 1992.

[22] Vasily E. Tarasov and George M. Zaslavsky. Fractional GinzburgLandau equation for fractal media. Physica A: Statistical Mechanics and its Applications, 354:249-261, August 2005.

[23] Hong Wang and Treena S. Basu. A fast finite difference method for two-dimensional space-fractional diffusion equations. SIAM Journal on Scientific Computing, 34(5):A2444-A2458, January 2012.

[24] Q. Xu and J. S. Hesthaven. Discontinuous galerkin method for fractional convection-diffusion equations. arXiv:1304.6047, April 2013.

[25] Ahmet Yldrm and Syed Tauseef Mohyud-Din. Analytical approach to space- and time-fractional burgers equations. Chinese Physics Letters, 27(9):090501, September 2010.

[26] Mohsen Zayernouri and George Em Karniadakis. Exponentially accurate spectral and spectral element methods for fractional ODEs. Journal of Computational Physics, 257, Part A:460-480, January 2014. 